\title{
Maxillary Orthodontic Expansion Assisted by Unilateral Alveolar Corticotomy and Low-Level Laser Therapy: A Novel Approach for Correction of a Posterior Unilateral Cross-Bite in Adults
}

\author{
Gianluigi Caccianiga', Antonino Lo Giudice ${ }^{2 *}$, Alessio Paiusco¹, Marco Portelli², Angela Militi², Marco \\ Baldoni $^{1}$, Riccardo Nucera ${ }^{2}$
}

${ }^{1}$ School of Medicine and Surgery, University of Milano-Bicocca, 20900 - Monza, Italy

${ }^{2}$ Department of Biomedical and Dental Sciences and Morphofunctional Imaging-Section of Orthodontics, School of Dentistry, University of Messina, 98124 - Messina, Italy

\author{
*Correspondence to \\ Antonino Lo Giudice, DDS, \\ MSc, PhD; Adjunct Professor \\ Department of Biomedical \\ and Dental Sciences and \\ Morphofunctional Imaging, \\ Section of Orthodontics, School of \\ Dentistry, University of Messina, \\ Policlinico Universitario "G. \\ Martino", Via Consolare Valeria - \\ 98123 Messina, Italy. \\ Tel: +390902216911 \\ Email: nino.logiudice@gmail.com
}

Published online July 6, 2019

\begin{abstract}
Introduction: The treatment of a true unilateral posterior crossbite often requires asymmetric maxillary expansion; however, this is challenging to achieve with conventional expansion methods because of several biomechanical limitations. In this paper, we introduce a new protocol for the treatment of a unilateral posterior crossbite in adults based on maxillary orthodontic expansion assisted by corticotomy and low-level laser therapy (LLLT) performed on the crossbite side.

Methods: The study sample included 15 adults ( 8 females, 7 males) affected by a true unilateral posterior crossbite, with a mean age of $21.6 \pm 3.1$ years at the at the beginning of treatment. After the application of orthodontic appliances (palatal expander and self-ligating brackets), corticomy was performed at the buccal aspect of the crossbite side while LLLT was monthly administered up to the correction of the crossbite. The efficacy of the technique was evaluated through measurements performed on maxillary digital models.

Results: All subjects reported successful correction of the posterior unilateral crossbite, and functional occlusion was achieved as well. The average expansion was greater at the crossbite side compared to the unaffected side and such difference was significant at the levels of first premolars $(P<0.05)$, second premolars $(P<0.05)$ and first molars $(P<0.05)$.

Conclusion: Orthodontic maxillary expansion assisted by unilateral corticotomy and LLLT was effective in the treatment of the true unilateral crossbite.

Keywords: Corticotomy; LLLT; Unilateral crossbite; Maxillary expansion.
\end{abstract}

\section{Introduction}

A posterior crossbite is one of the most frequent malocclusions occurring in both childhood and adulthood. ${ }^{1,2}$ It can appear both bilaterally or unilaterally, and in the latter case, it can be further classified as either functional or true. ${ }^{3}$ A true unilateral posterior crossbite is related to the asymmetric contraction of the palatal bone and/or the dentoalveolar process ${ }^{4}$ and is more challenging to treat as unilateral expansion is necessary. ${ }^{5}$ In this respect, over-expansion on the unaffected side $e^{6,7}$ is a common side effect which complicates the orthodontic bio-mechanics and extends the overall treatment time. Moreover, correction of the posterior crossbite in adult patients often requires significant buccal tipping of posterior teeth in order to camouflage the skeletal constriction, thus significantly increasing the risk of periodontal damage. ${ }^{8}$

Corticotomy is a surgical procedure based on the incisions of the cortical bone, without the removal of osseous material. ${ }^{9}$ It induces the "regional acceleratory phenomenon" (RAP), which allows for faster regeneration of the tissue involved in the surgical procedure by increasing osteoblast and osteoclast activity. ${ }^{10}$ During this period, a transitory and reversible osteopenia occurs, reducing biomechanical resistance to orthodontic movement. ${ }^{10}$ In this respect, maxillary expansion with unilateral corticotomy has been recently proposed as a valid method to obtain differential expansion in a more controlled way than conventional orthodontics in the treatment of a unilateral posterior crossbite, since tooth movement is expected to be enhanced more on the corticomized site than on the non-corticomized site. ${ }^{11}$

Please cite this article as follows: Caccianiga G, Lo Giudice A, Paiusco A, et al. Maxillary orthodontic expansion assisted by unilateral alveolar corticotomy and low-level laser therapy: a novel approach for correction of a posterior unilateral cross-bite in adults. J Lasers Med Sci. 2019;10(3):225-229. doi:10.15171/jlms.2019.36. 
In addition to the reduction of biomechanical resistance, the efficiency of orthodontic movement can be enhanced by stimulating the periodontal response to the application of orthodontic forces. In this respect, low-level laser therapy (LLLT) represents a non-invasive method of accelerating orthodontic tooth movement, that acts through different molecular and cellular mechanisms. ${ }^{12,13}$ LLLT has been shown to stimulate the growth and proliferation of osteoblasts and fibroblasts, to increase the levels of fibronectin and collagen-I levels and to increment RANK-L levels in the periodontal ligaments. ${ }^{12-14}$

The aim of the present study was to assess the effectiveness of a new technique in the correction of the unilateral posterior crossbite in adult patients, which is based on orthodontic expansion assisted by corticotomy and LLLT on the affected side. This technique combines the effect of lower bio-mechanical resistance induced by corticotomy and the biological accelerated response induced by LLLT in order to obtain greater expansion on the affected side compared to the unaffected side of subjects with a unilateral posterior crossbite.

\section{Methods}

This single operator, split-mouth designed, interventional prospective study was performed in the observance of the Declaration of Helsinki. Patients were treated between February 2013 and January 2018 at the Private Practice in Bergamo, Italy and all signed the appropriate informed consent.

\section{Subjects' Inclusion Criteria}

Patients were selected based on the following inclusion criteria: permanent dentition, posterior unilateral crossbite and cervical maturation stage CS6 $6^{15}$ as assessed from cephalograms. Exclusion criteria were: bilateral posterior crossbite or functional unilateral crossbite, dental agenesis, cranio-facial syndromes, and periodontal disease. The final study sample included fifteen patients (8 females and 7 males), with a mean age of $21.6 \pm 3.1$ years at the beginning of treatment. Figure 1 shows the occlusal characteristics of one female subject included in the present case series.

A power calculation was performed using a specific toolkit (DSS research, Washington, USA, https://www. dssresearch.com/knowledgecenter/toolkitcalculators/ samplesizecalculators.aspx) and it was indicated that 12 participants would yield a confidence level of $95 \%$ and a Beta error level of $85 \%$, thus it would be adequate to determine statistically significant differences. However, we decided to include 15 subjects to enhance the power of the study.

\section{Corticotomy Procedure}

The application of fixed orthodontic appliances and corticotomy was conducted to each patient on the same day (Figure 2). A Nitanium Palatal Expander 2 (NPE2, Ortho Organizer, Carlsbad, CA, USA) with bands on the first molars was applied to the upper arch along with self-ligating brackets (Empower; American Orthodontics, Sheboygan, WI, USA) bonded from right to left second premolars. Corticotomy was performed on the crossbite side immediately after the placement of orthodontic appliances. A preliminary evaluation of the sites of cortical incisions was performed on CT cone beam scans. Local anesthesia was administered on the buccal aspect of the affected side. A full thickness flap was raised in the area between the distal aspect of the upper first molar and the mesial aspect of the canine/lateral incisor. Vertical incisions in each interproximal space were performed and maintained apical to the interdental papilla. Cortical bone was cut for $3 \mathrm{~mm}$ of depth, assessed by a periodontal probe. Vertical corticotomies were connected by a
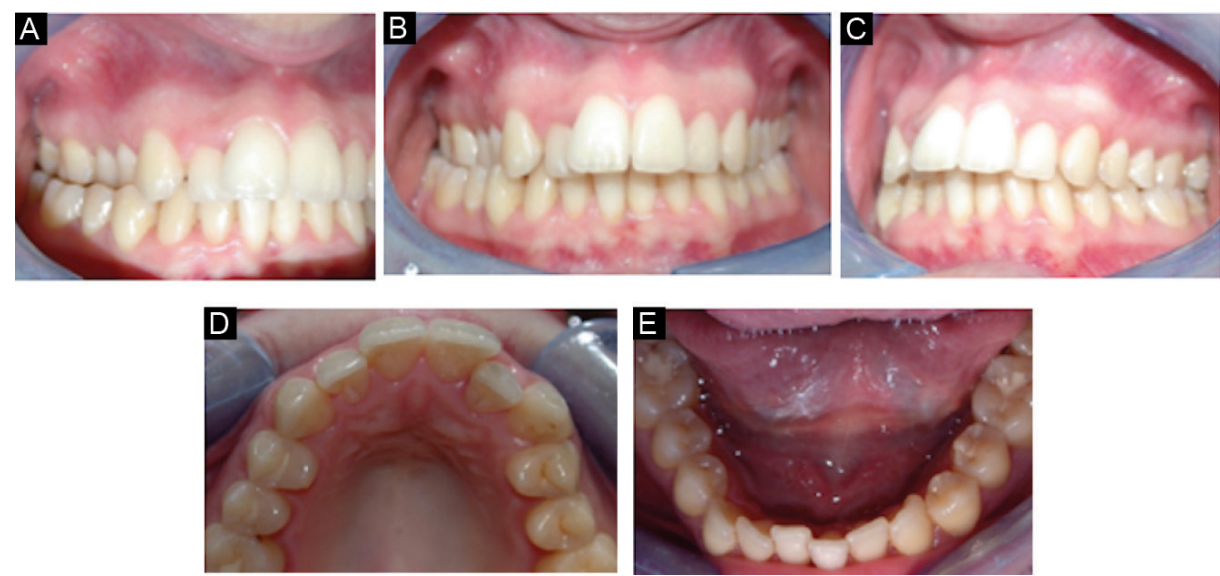

Figure 1. Occlusal Characteristics of One Female Subject Affected by a True Unilateral Posterior Crossbite. (A) Intraoral right lateral view (see the presence of the posterior crossbite), (B) Intraoral frontal view, (C) Intraoral left lateral view, (D) Intraoral upper occlusal view, (E) Intraoral lower occlusal view. 

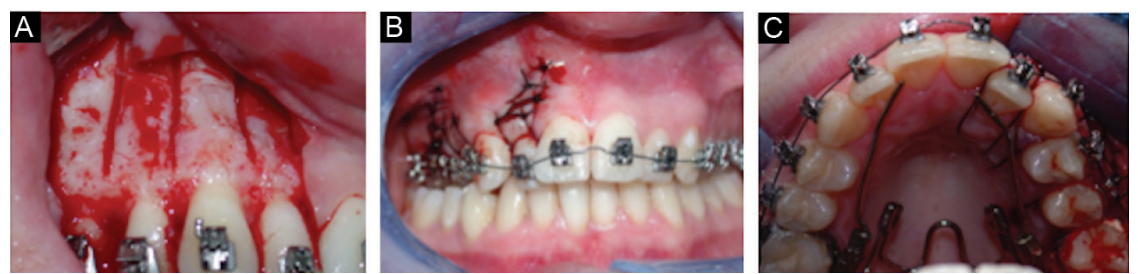

Figure 2. (A) Corticotomy incisions performed at the buccal aspect of the crossbite side. (B-C) Frontal and view after surgical procedure and installation of orthodontic appliances, (B) Self-ligating brackets, (C) Palatal expander.

horizontal corticotomy, at least $2 \mathrm{~mm}$ apical to the root apex of each tooth. Finally, the flap was sutured using a non-absorbable $4 / 0$ suture. In seven subjects ( 4 males, 3 females), corticotomy was carried out with a Piezotome (Implantcenter 2 Led, Satelec-Financiere Acteon SAS, Merignac), while in eight subjects (5 males, 3 females), the Erbium:Yag laser was used (Pluser, Doctor Smile - Lambda Spa, Brendola, VI), featuring the following characteristics: a wavelength of $2940 \mathrm{~nm}$, output of 0,18 J, average power of 3,6 W (0,18 J x $20 \mathrm{~Hz})$, energy density of $63,69 \mathrm{~J} / \mathrm{cm}^{2}$ and a 600 micron sapphire glass tip. The irradiation was performed using the beam as a pencil, in continuous motion. Meanwhile, a combination of $70 \%$ water and $90 \%$ air was used as a coolant agent in order to avoid bone overheating. Subjects were randomly allocated to receiving Piezotome or the Erbium:Yag laser by using a randomized balanced block protocol using sex as a stratification factor.

\section{Low-Level Laser Therapy Administration}

Once a month, all patients underwent LLLT at the crossbite side using a diode laser with a wavelength of $980 \mathrm{~nm}$ (Wiser, Doctor Smile - Lambda Spa, Brendola, VI) and power of 1 Watt set on the continuous wave mode. The beam was delivered by means of a plane wave optical fiber (AB 2799, Doctor Smile - Lambda Spa, Brendola, $\mathrm{VI}$ ), dispensing a beam spot size of $1 \mathrm{~cm}^{2}$ and irradiation was administered by positioning the optical fiber tip along the maxillary crossbite side $(1.5 \mathrm{~cm}$ as minimum on defocalization, as prescribed by the producer). Specifically, three dental segments were irradiated for 16 seconds (first and second molar), 11 seconds (first and second premolar) and 10 seconds (canine - lateral incisor) respectively, for a total of 37 seconds (Figure 1). The procedure was repeated 3 times at intervals of 2 minutes. All irradiations were done with output power of $1 \mathrm{~W}$ at a continuous wave. The total energy density for affected dental segment, corresponding to an exposure time of 111 seconds, was $111 \mathrm{~J} / \mathrm{cm}^{2}$ ( $1 \mathrm{~J} / \mathrm{cm}^{2}$ per second), including 48 $\mathrm{J} / \mathrm{cm}^{2}$ for the first molar-second molar segment, $33 \mathrm{~J} / \mathrm{cm}^{2}$ for the first premolar-second premolar segment and $30 \mathrm{~J} /$ $\mathrm{cm}^{2}$ for the first canine-lateral incisor segment. ${ }^{13,14}$

Assessment of Pre-treatment/Post-treatment Maxillary Transversal Changes

The efficacy of the technique was evaluated through the analysis of digital models of the maxillary arch acquired before and after completion of orthodontic treatment. In particular, distances between the maxillary midline and canine, first and second premolars and first molars were calculated on both crossbite and non-crossbite sides, using both dental and gingival landmarks according to Leonardi et al. ${ }^{4}$ Differences between the measurements obtained before and after treatment were determined. All measurements were recorded on Microsoft Excell ${ }^{\circledR}$ spreadsheet (Microsoft, Redmond WA, USA) and analyzed using SPSS ${ }^{\circledR}$ version 24 Statistics software (IBM Corporation, 1 New Orchard Road, Armonk, New York, USA). $P$ values of less than 0.05 were considered statistically significant. Normal distribution of data was preliminary checked by using the Kolmogorov-Smirnov test; since data were not normally distributed, variation between pre- and post-treatment measurements was calculated and assessed using one-tailed Wilcoxon signed rank test with the significance level set at $P<0.05$.

\section{Results}

All subjects included in the present study reported successful correction of the posterior unilateral crossbite. In particular, the average expansion at the crossbite side was greater than that observed at the non-crossbite side in each patient (Table 1). In this respect, such differential expansion was significant at first premolars $(P<0.05)$, second premolars $(P<0.05)$ and first molars $(P<0.05)$ levels considering both dental and gingival landmark analyses.

\section{Discussion}

The successful treatment of a true unilateral posterior crossbite often requires asymmetric maxillary expansion, especially in those cases presenting emilateral transversal maxillary deficiency. However, unilateral maxillary expansion is challenging to achieve since palatal expanders distribute forces in a symmetrical pattern; despite the fact that several designs and modifications have been proposed, over-expansion is a common side effect, ${ }^{6,7}$ which complicates the orthodontic biomechanics, extends the treatment time and increases the risk of periodontal damage. ${ }^{8}$ Mini-screw placed on the palatal has been recently suggested for asymmetric transverse control of maxillary dentition ${ }^{16}$; however, the risk of failure associated with TADs can represent the 
Table 1. Differences between pre-treatment (T1) and post-treatment (T2) unilateral expansion calculated both at the crossbite side (corticotomy YES) and the non-crossbite side (corticotomy NO)

\begin{tabular}{|c|c|c|c|c|c|}
\hline \multirow{2}{*}{ Landmarks } & \multirow{2}{*}{ Patients } & $\begin{array}{c}\text { Crossbite Side } \\
\text { (Corticotomy Tes) }\end{array}$ & $\begin{array}{c}\text { Non-crossbite Side } \\
\text { (Corticotomy No) }\end{array}$ & \multirow[b]{2}{*}{$P$ Value } & \multirow[b]{2}{*}{ Significance } \\
\hline & & $\begin{array}{l}\mathrm{T} 2-\mathrm{T} 1(\%) \\
\text { mean } \pm \mathrm{SD}\end{array}$ & $\begin{array}{l}\mathrm{T} 2-\mathrm{T} 1(\%) \\
\text { mean } \pm \mathrm{SD}\end{array}$ & & \\
\hline \multicolumn{6}{|c|}{ Dental Landmarks } \\
\hline U6-MPP & 15 & $6.45 \pm 1.90$ & $2.98 \pm 3.87$ & 0.02 & S \\
\hline U5-MPP & 15 & $8.78 \pm 1.51$ & $3.32 \pm 1.79$ & 0.03 & S \\
\hline U4-MPP & 15 & $7.83 \pm 2.34$ & $3.65 \pm 2.21$ & 0.01 & S \\
\hline U3-MPP & 15 & $4.01 \pm 3.08$ & $2.94 \pm 1.93$ & 0.47 & NS \\
\hline \multicolumn{6}{|c|}{ Gingival Landmarks } \\
\hline U6-MPP & 15 & $4.19 \pm 0.72$ & $1.87 \pm 1.56$ & 0.01 & S \\
\hline U5-MPP & 15 & $6.31 \pm 3.09$ & $2.25 \pm 2.19$ & 0.02 & $S$ \\
\hline U4-MPP & 15 & $5.99 \pm 3.11$ & $2.82 \pm 2.36$ & 0.03 & S \\
\hline U3-MPP & 15 & $3.20 \pm 2.93$ & $1.98 \pm 2.03$ & 0.34 & NS \\
\hline
\end{tabular}

main limitation for this purpose.

In the present study, we tested the effectiveness of maxillary expansion combined with corticotomy and LLLT at the buccal aspect of the crossbite side for the treatment of the unilateral posterior crossbite. We obtained complete correction of this malocclusion in all treated subjects, restoring the physiological dental contacts in centric occlusion and avoiding significant over-expansion on the unaffected side.

It can be postulated that both the RAP and the lower bone density induced by corticotomy have favoured a greater expansion on the crossbite side, as previously suggested by Hassan et al. ${ }^{11}$ However, the authors performed a more invasive procedure including corticotomy both at the buccal and palatal aspects of the crossbite side and at the buccal aspect of the unaffected side. Based on our findings, corticotomy should be performed on the affected side since expansion is required in this area, and should be limited to the buccal aspect of the alveolar process in order to reduce only that bone resistance preventing the desired tooth position (i.e., lateral expansion).

In the present study, the greater expansion obtained on the crossbite side could also be attributed to the accelerated periodontal response induced by LLLT. ${ }^{12,13,17}$ In this respect, it has been demonstrated that LLLT accelerates cellular turnover by increasing vascular activity and the availability of ATP, stimulates proliferation of osteoblasts, increases fibronectin and collagen I levels, and stimulates fibroblast growth and proliferation. ${ }^{12-14,18,19}$ In the orthodontic field, the assumption is that such increased cellular metabolic activity can speed the rate of bone remodeling, as recently demonstrated by clinical trials. $^{12,17}$

We installed the palatal expander on the same day of the surgical procedure, thus no latency period was planned between corticotomy and the beginning of orthodontic therapy. This could have facilitated the expansion on the crossbite side according to previous studies suggesting the immediate application of orthodontic forces in order to guarantee adequate tooth movement prior to the beginning of the osteoblastic activity. ${ }^{20,21} \mathrm{New}$ bone formation is known to begin 2-4 weeks after corticotomy is performed and such a phenomenon increases resistance to tooth movement. ${ }^{20,21}$

The efficacy of corticotomy-assisted maxillary expansion depends on the entity of RAP which, in turn, is proportional to the trauma induced by corticotomy. ${ }^{22}$ In the present study, 8 patients were treated using the Erbium:Yaglaser which is less traumatic than piezosurgery or a carbide bur. ${ }^{23-24}$ All subjects reached sufficient expansion for the correction of the unilateral crossbite, suggesting that Erbium:Yag is as effective as piezosurgery in performing corticotomy. We observed that the Erbium:Yag laser was more comfortable for patients, given the lack of vibrations, ${ }^{23-25}$ the absence of sub-cutaneous hematomas and less post-operative pain compared to the use of piezosurgery. The use of Erbium:Yag laser is also suggested for flapless surgery. Nevertheless, corticotomy should require flap preparation to obtain direct visual access to dental roots, avoiding the risk of trauma, unless computer-guided flapless corticotomy ${ }^{26}$ is performed, which, in turn, is more expensive and time-consuming.

It must be underlined that the present findings are based on limited sample size; thus, further prospective studies with greater sample size are required in order to elucidate the efficacy of the combined use of orthodontic maxillary expansion, corticotomy and LLLT for the treatment of the posterior unilateral cross-bite in adults.

\section{Conclusion}

Orthodontic maxillary expansion assisted by unilateral corticotomy and LLLT was effective in the treatment of the true unilateral crossbite. This technique is relatively more invasive than conventional expansion methods due to corticotomy incisions. However, it is less invasive than surgical-assisted maxillary expansion and could be 
considered a valid alternative to the treatment of moderate to severe unilateral transversal maxillary deficiency.

\section{Ethical Considerations}

This clinical trial was approved by the ethics committee of the Faculty of Medicine, Milano-Bicocca University.

\section{Conflict of Interests}

The authors declare no conflict of interest.

\section{References}

1. Binder ER. Correction of posterior crossbites: diagnosis and treatment. Pediatr Dent. 2004;26:266-272.

2. Posnick JC. Principles and Practice of Orthognatic Surgery. Elsevier Health Sciences; 2013:271.

3. Proffit WR, Fields HW, Sarver DM. Contemporary Orthodontics. Elsevier Health Sciences; 2014:699.

4. Leonardi R, Lo Giudice A, Rugeri M, Muraglie S, Cordasco G, Barbato E. Three-dimensional evaluation on digital casts of maxillary palatal size and morphology in patients with functional posterior crossbite. Eur J Orthod. $2018 \mathrm{Feb}$ 21. doi: $10.1093 /$ ejo/cjx103

5. Ileri Z, Basciftci FA. Asymmetric rapid maxillary expansion in true unilateral crossbite malocclusion: a prospective controlled clinical study. Angle Orthod. 2015;85(2):245-52. doi:10.2319/011214-40.

6. Nerde, PH, Bakke M, Solow B. The functional shift of the mandible in unilateral posterior crossbite and the adaptation of the temporomandibular joints: a pilot study. Eur J Orthod 1999;21:155-166.

7. Brin I, Ben-Bassat Y, Blustein Y, et al. Skeletal and functional effects of treatment for unilateral posterior crossbite. Am J Orthod Dentofacial Orthop. 1996;109:173-179.

8. Vanarsdall RL Jr. Transverse dimension and long-term stability. Semin Orthod. 1999;5:171-180.

9. Verna C, Cattaneo PM, Dalstra M. Corticotomy affects both the modus and magnitude of orthodontic tooth movement. Eur J Orthod. 2018;40(1):107-112. doi: 10.1093/ejo/cjx041.

10. Frost HM. The regional acceleratory phenomenon: a review. Henry Ford Hosp Med J. 1983;31(1):3-9.

11. Hassan AH; Al-Fraidi AA, Al-Hubail A, Hajrassy MK. Unilateral cross bite treated by corticotomy-assisted expansion: two case reports. Head Face Med. 2010;6:6.

12. Caccianiga G, Paiusco A, Perillo L, et al. Does low-level laser therapy enhance the efficiency of orthodontic dental alignment? Results from a randomized pilot study. Photomed Laser Surg. 2017;35(8):421-426.

13. Caccianiga G, Crestale C, Cozzani M, Piras A, Mutinelli S, Lo Giudice A, Cordasco G. Low level laser therapy and invisible removal aligners. J Biol Regul Homeost Agents. 2016;30(2):107-113.

14. Leonida A, Paiusco A, Rossi G, Carini F, Baldoni M, Caccianiga G. Effects of low-level laser irradiation on proliferation and osteoblastic differentiation of human mesenchymal stem cells seeded on a three- dimensional biomatrix: In vitro pilot study. Lasers Med Sci. 2013;28(1):125-132. doi:10.1007/s10103-012-1067-6

15. Baccetti T, Franchi L, McNamara JA Jr. An improved version of the cervical vertebral maturation (CVM) method for the assessment of mandibular growth. Angle Orthod. 2002;72(4):316-323.

16. Kwon HY, Mah SJ, Kang YG. Asymmetric transverse control of maxillary dentition with two midpalatal orthodontic miniscrews. Angle Orthod. 2015;85(3):525534. doi:10.2319/042514-302.1.

17. Qamruddin I, Alam MK, Mahroof V, Fida M, Khamis MF, Husein, A. Effects of low-level laser irradiation on the rate of orthodontic tooth movement and associated pain with self-ligating brackets. Am J Orthod Dentofacial Orthop. 2017;152(5):622-630.

18. Grassi FR, Ciccolella F, D'Apolito G, et al. Effect of lowlevel laser irradiation on osteoblast proliferation and bone formation. J Biol Regul Homeost Agents. 2011;25(4):603614.

19. Lo Giudice A, Nucera R, Perillo L, Paiusco A, Caccianiga G. Is low-level laser therapy an effective method to alleviate pain induced by active orthodontic alignment archwire? a randomized clinical trial. J Evid Based Dent Pract. 2019;19(1):71-78.

20. Suya H. Corticotomy in orthodontics. In: Hosl E. Baldauf A, eds. Mechanical and biological basis in orthodontic therapy. Heidelberg: Huthing Buch Verlag; 1991:207-226.

21. Gantes B, Rathbun E, Anholm M. Effects on the periodontum following corticotomy-facilitated orthodontics. Case reports. J Periodontol. 1990 Apr;61(4):234-8. doi:10.1902/ jop.1990.61.4.234

22. Cohen G, Campbell PM, Rossouw PE, Buschang PH. Effects of increased surgical trauma on rates of tooth movement and aplical root resorption in foxhound dogs. Orthod Craniofac Res. 2010;13(3):179-90. doi: 10.1111/j.16016343.2010.01494.x

23. Caccianiga G, Baldoni M, Ghisalberti CA, Paiusco A. A preliminary in vitro study on the efficacy of high-power photodynamic therapy (HLLT): comparison between pulsed diode lasers and superpulsed diode lasers and impact of hydrogen peroxide with controlled stabilization. Biomed Res Int. 2016;2016:1386158. doi: 10.1155/2016/1386158

24. Inchingolo F, Tatullo M, Abenavoli FM, et al. Comparison between traditional surgery, $\mathrm{CO} 2$ and $\mathrm{Nd}$ :Yag laser treatment for generalized gingival hyperplasia in SturgeWeber syndrome: a retrospective study. J Investig Clin Dent. 2010;1(2):85-9. doi:10.1111/j.2041-1626.2010.00020.x

25. De Benedittis $\mathrm{M}$, Petruzzi M, Pastore L, Inchingolo $\mathrm{F}$, Serpico R. Nd:YAG laser for gingivectomy in Sturge-Weber syndrome. J Oral Maxillofac Surg. 2007;65(2):314-316. doi:10.1016/j.joms.2006.05.011

26. Cassetta M, Altieri F, Pandolfi S, Giansanti M. The combined use of computer-guided, minimally invasive, flapless corticotomy and clear aligners as a novel approach to moderate crowding: A case report. Korean J Orthod. 2017;47(2):130-141. doi:10.4041/kjod.2017.47.2.130 